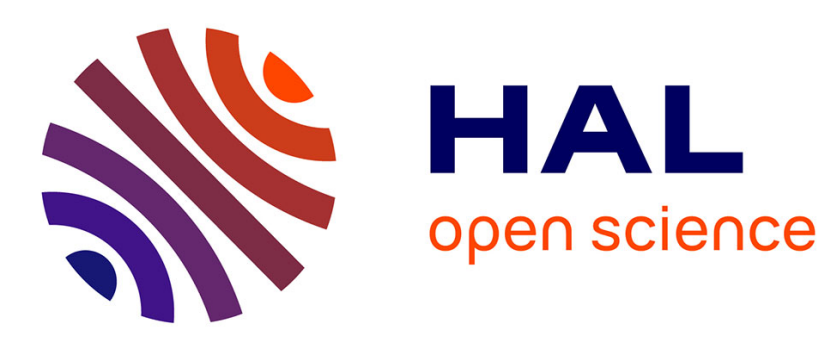

\title{
New Methods for a Fast and Easy Computation of Stray Fields Created by Wound Rods
}

\author{
Olivier Chadebec, Laure-Line Rouve, Jean-Louis Coulomb
}

\section{To cite this version:}

Olivier Chadebec, Laure-Line Rouve, Jean-Louis Coulomb. New Methods for a Fast and Easy Computation of Stray Fields Created by Wound Rods. IEEE tansaction on magnetics, 2002, 38 (2), pp.517-520. 10.1109/20.996136 . hal-00289974

\section{HAL Id: hal-00289974 \\ https://hal.science/hal-00289974}

Submitted on 24 Jun 2008

HAL is a multi-disciplinary open access archive for the deposit and dissemination of scientific research documents, whether they are published or not. The documents may come from teaching and research institutions in France or abroad, or from public or private research centers.
L'archive ouverte pluridisciplinaire HAL, est destinée au dépôt et à la diffusion de documents scientifiques de niveau recherche, publiés ou non, émanant des établissements d'enseignement et de recherche français ou étrangers, des laboratoires publics ou privés. 


\title{
New Methods for a Fast and Easy Computation of Stray Fields Created by Wound Rods
}

\author{
Olivier Chadebec, Laure-Line Rouve, and Jean-Louis Coulomb
}

\begin{abstract}
A quick and easy computation involving magnetic rods is presented. Rods are replaced by different kinds of distributions (magnetization, magnetic charges) calculated by collocation. These distributions can be punctual or surfacic (disc) in the rod according to the density of the mesh used. Then, three-dimensional geometries can be quickly and easily modeled instead of using uneasy finite-element methods.
\end{abstract}

Index Terms-Boundary element method, line elements, magnetostatics, moment method, wound rods.

\section{INTRODUCTION}

$\mathbf{S}$ OME magneto torque bars are used in order to correct satellite trajectory with respect to earth field direction. A magneto torque bar (MTB) consists of a magnetic rod around which a coil is wound. On board a satellite, these MTB create radiated magnetic fields that can be a disturbance for other MTB (and other structures). This is why it is necessary to compute the magnetic interaction between MTB (three-dimensional (3-D) problem).

A classical 3-D finite-element method can be used but the mesh of such rods (small diameter, high length) leads rapidly to a huge matrix system which is time and memory consuming to solve. A way to bypass the problem is to use line region for the rods [1]. But, systems still have a big size (the surrounding air volume has to be meshed).

We propose an approach based on moment method. We will see that the a magnetized rod can be replaced by a distribution of moments (i.e., magnetization) This moment distribution is mathematically equivalent to a charge distribution. In a first step, the distribution is condensed at different points on a line representative of the rod. Two formulations are explained to obtain them. Once distributions have been obtained, it is then easy to calculate the field everywhere in the surrounding air region. In a second step, we will see that these approaches lead to inaccuracies for fine meshes (this kind of mesh is necessary to take into account quick variations of inductor field created by winding). The condensation on a line is then replaced by constant distributions on cylinders, equivalent to constant distribution of charges on discs. Results obtained are then improved. Finally, an example of 3-D modeling is shown.

Manuscript received July 5, 2001; revised October 25, 2001

O. Chadebec and L.-L. Rouve are with the Laboratoire du Magnétisme du Navire, 38402 Saint Martin d'Hères, France (e-mail: chadebec@leg.ensieg.inpg.fr).

J.-L. Coulomb is with the Laboratoire d'Electrotechnique de Grenoble, 38402 St Martin d'Hères, France (e-mail: coulomb@leg.ensieg.inpg.fr).

Publisher Item Identifier S 0018-9464(02)02520-7.

\section{LINE FORMULATION}

Let us consider a linear magnetic law for the material

$$
\mathbf{M}(P)=\left(\mu_{r}-1\right) \mathbf{H}(P)
$$

where $\mathbf{M}(P)$ is the magnetization $(\mathrm{A} / \mathrm{m}), \mathbf{H}(P)$ is the local magnetic field $(\mathrm{A} / \mathrm{m})$ and $\mu_{r}$ is the relative permeability of material at the point $P$. The induced magnetization of the rod creates a local perturbation of the field, which can be expressed in terms of reduced field $\mathbf{H}_{\text {red }}$

$$
\mathbf{H}=\mathbf{H}_{\mathbf{0}}+\mathbf{H}_{\text {red }} .
$$

The reduced field at one point of the material is created by the magnetization of all the rod. Its expression is [2]

$$
\mathbf{H}_{\text {red }}(P)=-\frac{1}{4 \pi} \int_{V} \operatorname{grad}\left(\mathbf{M}(Q) \cdot \frac{\mathbf{r}}{r^{3}}\right) d V
$$

where $V$ is the volume of the rod and $\mathbf{r}$ the vector between a point of the $\operatorname{rod} Q$ and the point of calculus $P$ of the reduced field.

As the ratio rod length/rod diameter and the permeability material are high, flux lines are assumed parallel to the rod axis and magnetization uniform along the section [1]. As a consequence, the rod can be reduced to a line. Equation (3) becomes then a line integration and by combining it with (2) and (1), we obtain

$$
\begin{aligned}
\mathbf{M}(P)+\frac{s\left(\mu_{r}-1\right)}{4 \pi} \int_{L} \operatorname{grad}\left(\mathbf{M}(Q) \cdot \frac{\mathrm{r}}{r^{3}}\right) d L \\
=\left(\mu_{r}-1\right) \mathbf{H}_{\mathbf{0}}(P)
\end{aligned}
$$

where $s$ is the rod section.

Magnetization can also be represented by a distribution of charges [3]. The two distributions are linked by

$$
\rho=-\operatorname{div} \mathbf{M} \text {. }
$$

As the magnetization is constant on the section, (5) can be simplified by

$$
\rho=-\operatorname{div}_{L} \mathbf{M}
$$

where $\operatorname{div}_{L}$ is the standard divergence applied only along the rod axis. The charge distribution is then proportional to the derivative of the magnetization. By combining (4) and (6), we obtain a global equation for charges distribution

$$
\begin{aligned}
-\rho(P)-\frac{s\left(\mu_{r}-1\right)}{4 \pi} \operatorname{div}_{L} \int_{L} \rho(Q) \frac{\mathbf{r}}{r^{3}} d L \\
=\left(\mu_{r}-1\right) \operatorname{div}_{L} \mathbf{H}_{\mathbf{0}}(P) .
\end{aligned}
$$


Equations (4) and (7) are singular for $Q=P$. It is the principal difficulty for their resolution. They have already been obtain in [4] for thin shell structure.

\section{CONDENSATION OF DistRIBUTIONS}

In this section, we present some very quick and easy methods to solve (4) and (7).

\section{A. Constant Magnetization Distribution on a Line}

Let us consider a single rod meshed into $N$ line elements. Magnetization is assumed to be uniform along the line element $i$ and its value is $M_{i}$. By applying a collocation method to (4) at the center of each element, it comes

$$
\mathbf{M}_{i}=\left(\mu_{r}-1\right)\left(\mathbf{H}_{0 i}-\frac{s}{4 \pi} \sum_{j=1, N} \int_{L_{j}} \operatorname{grad} \frac{\mathbf{M}_{j} \cdot \mathbf{r}_{j i}}{r_{j i}^{3}} d L_{j}\right)
$$

where $\mathbf{r}_{j i}$ is the vector from the current point on element $j$ to the center of element $i$ and $\mathbf{H}_{\mathbf{0}}$ is the external field at this point. This vectorial equation can be projected along the axis of the line $L$. Thus, it becomes a scalar expression which is singular for $i=j$.

To reduce this singularity, a classical result is applied on each element. Let us consider a volume $V$, delimited by the surface $S$, with a uniform magnetization. Mathematically, the field created by the volume distribution of $\mathbf{M}$ is equivalent to a distribution of charges located on $S$

$$
\begin{aligned}
\mathbf{H}_{\text {red }} & =-\frac{1}{4 \pi} \int_{V} \operatorname{grad}\left(\mathbf{M}(Q) \cdot \frac{\mathbf{r}}{r^{3}}\right) d V \\
& =-\frac{1}{4 \pi} \iint_{S} \mathbf{M} \cdot \mathbf{r} \frac{\mathbf{r}}{r^{3}} d S
\end{aligned}
$$

where $\mathbf{n}$ is the external normal to $V$. The surface charges distribution is equal to M.n. By applying (9) on each element $L_{i}$, we obtain a new expression for (8)

$$
\begin{aligned}
\mathbf{M}_{i}=\left(\mu_{r}-1\right) & \left(\mathbf{H}_{0 i}-\frac{s}{4 \pi} \sum_{j=1, N}\right. \\
& \cdot\left(\frac{\mathbf{M}_{j} \cdot \mathbf{n}_{1 j}}{r 1_{j i}^{3}} \mathbf{r}_{1 j i}+\frac{\mathbf{M}_{j} \cdot \mathbf{n}_{2 j}}{r 2_{j i}^{3}} \mathbf{r}_{2 j i}\right)
\end{aligned}
$$

where $\mathbf{n}_{1 j}, \mathbf{n}_{2 j}$ are the external normals of the element $j$ and $\mathbf{r}_{1 j i}, \mathbf{r}_{2 j i}$ are the vectors from the extremities of element $j$ to the center of element $i$.

Equation (10) represents a well known expression of the equivalence between a uniformly magnetized line and two associated punctual magnetic charges $+q,-q$ located at the line extremities, with (see Fig. 1)

$$
q=\text { (M.n) } s .
$$

Equation (8) does not present singularity anymore because the collocation point is at the center of the element and the integration point is located on the mesh nodes.

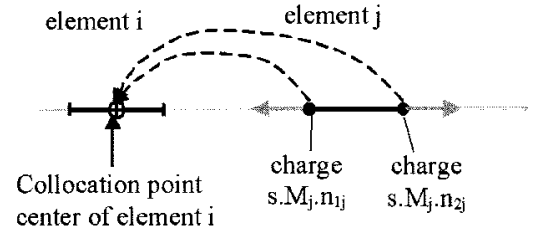

Fig. 1. Representation of (10).

By writing (10) for each element, a full linear system of $(N \times$ $N$ ) is built where the unknowns are the constant magnetizations $\mathbf{M}_{i}$ on each element. As we said, this distribution is strictly equivalent to a punctual distribution of charge located on each node of the mesh. Once the system has been solved, the induction can then be calculated everywhere in the air region with

$$
\mathbf{B}=\mu_{0}\left(\mathbf{H}_{0}-\frac{s}{4 \pi} \sum_{j=1, N}\left(\frac{\mathbf{M}_{j} \cdot \mathbf{n}_{1 j}}{r 1_{j i}^{3}} \mathbf{r}_{1 j i}+\frac{\mathbf{M}_{j} \cdot \mathbf{n}_{2 j}}{r 2_{j i}^{3}} \mathbf{r}_{2 j i}\right)\right. \text {. }
$$

\section{B. Punctual Charge Distribution}

It is possible to solve (7) directly by a global equation that describes flux equilibrium through a thin shell. This approach is presented in [4] and can be easily applied for a line geometry. We consider that charges are located at each nodes of the mesh. The equation obtained is then

$$
\begin{aligned}
-q_{i}=s\left(\mu_{r}-1\right)\left(\frac{1}{4 \pi} \sum_{j=1, N+1} q_{j} \int_{L_{i}} \frac{\mathbf{r}_{\mathbf{i j}}}{r_{i j}^{3}} \mathbf{n}_{i} d L\right. \\
\\
\left.\quad+\int_{L_{i}} \mathbf{H}_{\mathbf{O S}} \cdot \mathbf{n}_{i} d L\right) .
\end{aligned}
$$

A full linear system of $(N+1) \times(N+1)$ dimension is obtained. The unknowns are the condensed charges $q_{i}$ at each node. The induction in the air region is then

$$
\mathbf{B}=\mu_{0}\left(\mathbf{H}_{0}-\frac{1}{4 \pi} \sum_{j=1, N+1} q_{j} \frac{\mathbf{r}_{j}}{r_{j}^{3}}\right) .
$$

\section{Validation Test Case}

Our approach has been tested on a single rod of $270 \mathrm{~mm}$ length. Its diameter is $4.4 \mathrm{~mm}$ and its relative permeability is 100. To simplify, the rod is subjected to a uniform axial field of $3900 \mathrm{~A} / \mathrm{m}$.

A finite-element method is used to obtain a reference computation. It is a two dimension problem because of the simple studied geometry. The rod is meshed by volume elements (four elements in the section).

In a first step, the validation is based on the induction value along the rod axis calculated by volume finite-element method and by the resolution of (10) (i.e., constant magnetizations along a line). The rod is meshed into 27 line elements and results have good accuracy (see Fig. 2).

The same problem is solved thanks to (13). A punctual charge distribution is determined at each node of the mesh. This charge 


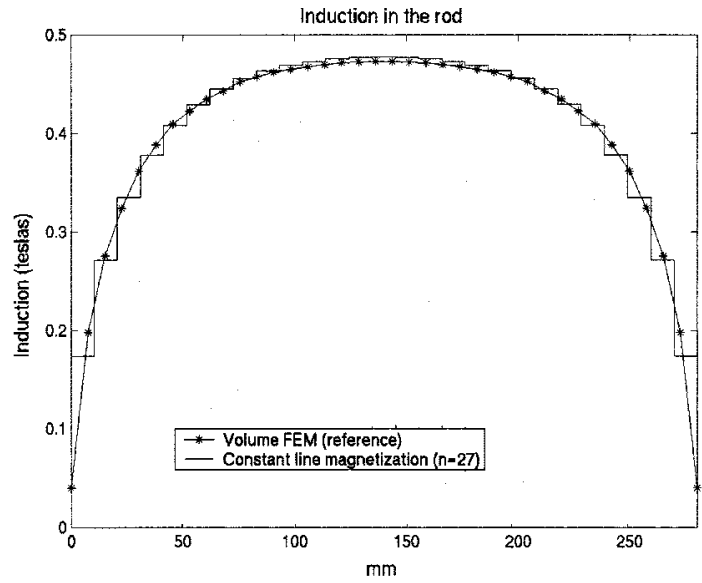

Fig. 2. Comparison between volume FEM and constant magnetization collocation $(N=27)$.

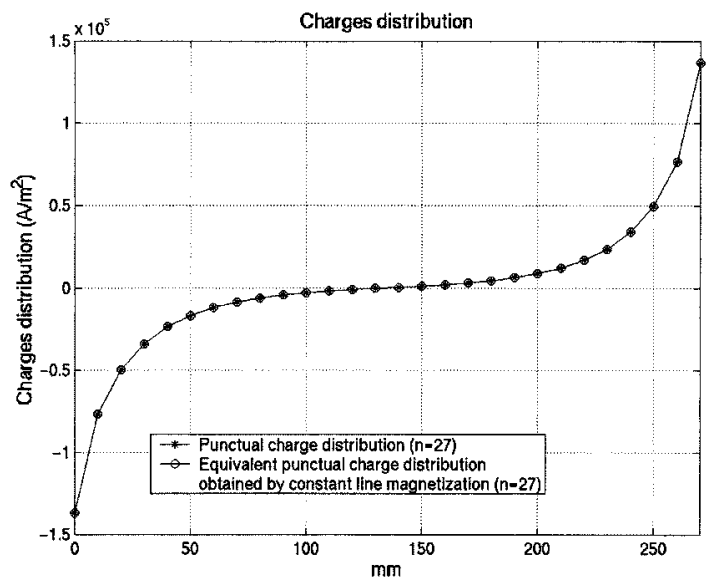

Fig. 3. Charge distributions along the rod obtained by the resolution of system (13) and by the one with of (10) followed by (11).

distribution can be compared to the charge distribution obtained by applying (11) to the previous magnetization modeling. Both distributions are equivalent (see Fig. 3). Let us notice that this distribution is proportional to the derivative of the induction.

\section{Conclusion on the Line Condensation}

Our two algorithms have been successfully validated for the simple case of a single rod placed in an external constant field. Both method are really easy to compute and leads to very small system $(27 \times 27$ for magnetization and $28 \times 28$ for charge distribution). The induction is predicted with a very good accuracy in comparison of a volume FEM of 10000 elements.

In this approach, a good accordance was expected with a higher discretization because the hypothesis "magnetization uniform along one line element" is better verified. However, when increasing the number of elements $N$ (216 for example), our methods present a discrepancy with respect to the FEM reference. This point has to be improved if we want to solve problems where magnetization along the rod varies a lot. It is especially the case when the rod is wound: the inductor field is no more uniform and can rapidly vary. In these cases, the rod must be highly discretized to correctly take into account magnetization variations.

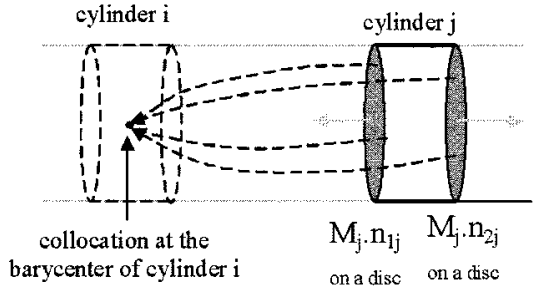

Fig. 4. Representation of (15).

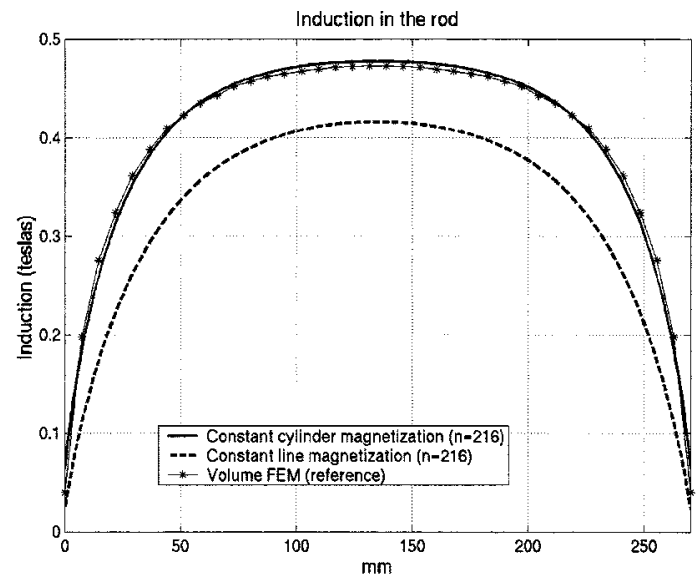

Fig. 5. Comparison between volume FEM, constant line magnetization collocation and constant cylinder magnetization collocation $(N=216)$.

\section{IMPROVEMENT OF THE INTEGRATION-CONSTANT MAGNETIZATION IN A CYLINDER}

For $N=27$, element length $(10 \mathrm{~mm})$ is of the same order as the rod diameter. When $N$ increases, element length becomes really smaller than the rod diameter. As a consequence, for a given element, it seems more accurate to compute the field by associating two uniformly charged discs [2] instead of two point charges to one element. The rod is then no more represented by uniformly magnetized segments but by uniformly magnetized cylinders. By applying (9), these uniformly magnetized cylinders are equivalent to two uniform charge discs located at each cylinder extremity (see Fig. 4). Integration term becomes the reduced field created by these discs at the center of each element

$$
\begin{gathered}
\mathbf{M}_{i}=\left(\mu_{r}-1\right)\left(\mathbf{H}_{0 i}-\frac{s}{4 \pi} \sum_{j=1, N} \frac{M_{j}}{2}\left(\frac{-\mathbf{r}_{1 j i}}{\sqrt{r_{1 j i}^{2}+a^{2}}}+\frac{\mathbf{r}_{1 j i}}{r_{1 j i}}\right)\right. \\
+\frac{s}{4 \pi} \sum_{j=1, N} \frac{M_{j}}{2}\left(\frac{-\mathbf{r}_{2 j i}}{\sqrt{r_{2 j i}^{2}+a^{2}}}+\frac{\mathbf{r}_{2 j i}}{r_{2 j i}}\right)
\end{gathered}
$$

where $a$ is the rod radius.

The resolution of system (15) is tested on the same rod as in the previous section but with a 216 element mesh. On the one hand, the induction computed with the line constant magnetization collocation leads to inaccuracies for coarse mesh. On the other hand, the new integration significantly improves the result. The induction along the rod is then calculated with a good accuracy with respect to volume FEM (see Fig. 5).

With this new integration, it is now possible to take into account quick variations of the external field on the rod. In pecu- 


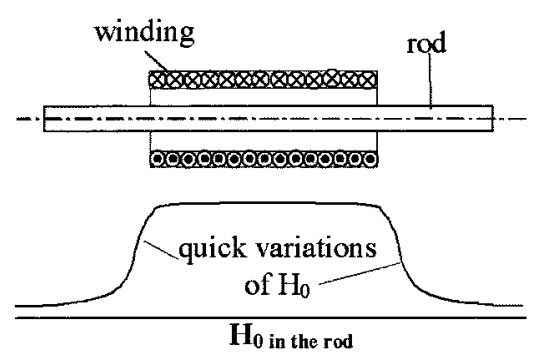

Fig. 6. Partially wound rod and the variations of the inductor field created by the winding require a fine mesh.

TABLE I

COMPARISON WITH DIFFERENT PERMEABILITIES

\begin{tabular}{cc} 
Relative Permeability & $\begin{array}{r}\text { Percent of difference } \\
\text { with FEM reference }\end{array}$ \\
\hline 10 & $1.1 \%$ \\
\hline 100 & $1 \%$ \\
\hline 1000 & $10.3 \%$ \\
\hline 10000 & winding \\
\hline
\end{tabular}

Fig. 7. Three-dimensional modeling of three MTB.

liar, partially wound rods can be modeled. This kind of MTB is not wound along the whole rod as shown on Fig. 6.

\section{Permeability VARiation}

For MTB, depending on the geometry, strong relative permeabilities can be used in order to have a linear law between winding current and the induced magnetic moment. In this section, the validity of our model with constant magnetization in cylinders is tested with permeability variations. The studied case is still the same, four different permeabilities are used. The reference result is still a volume finite-element modeling of the rod. We present here a comparison of maximal induction in the rod between FEM and constant magnetization cylinder approach in percent (see Table I).

Our approach leads to inaccuracies when modeling rods with high permeability. However, these inaccuracies are also obtained with line elements in FEM. We think that the approximation of tangential field in the rod is not verified in elements located at the extremities of the structure (the flux, there, is not well channeled by the rod). Nevertheless, the errors does not exceed $10 \%$, which can be satisfactory in compensation for a really fast and easy computation.

\section{EXAMPLE OF APPLICATION}

We now consider three MTB oriented in the three directions of the space (see Fig. 7).

The goal of the study is to know how an active MTB (with current inside its winding), magnetically interacts with the two

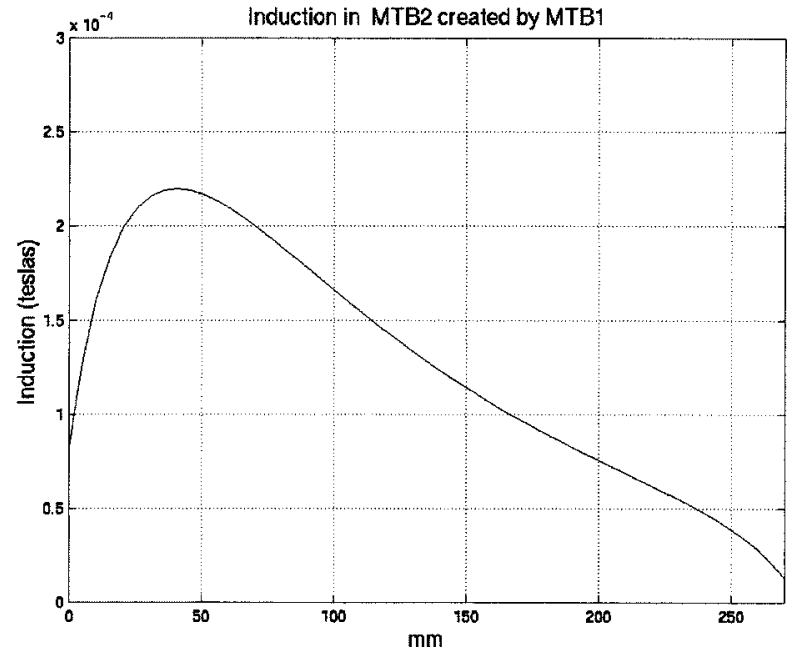

Fig. 8. Magnetization in MTB2 induced by active MTB1.

other passive MTB (no current inside their windings). In peculiar, magnetization induced in passive MTB must be determined.

The three MTB have the same characteristics as in Section III-C. The inductor field, created by winding of MTB1 is computed by Biot et Savart's Law. Ampere turns are equal to 528. Each MTB is meshed into 54 elements.

Interactions between MTB are taken into account as follows. Let us consider the magnetization $M_{i}$ of element $i$. To evaluate the influence of element $j$ (magnetization $M_{j}$ ), two cases are encountered. If $M_{i}$ and $M_{j}$ belong to the same MTB, integration as in (15) is used (charge disc distribution). If $M_{i}$ and $M_{j}$ belong to different MTB, integration similar to (10) is used. Thus, a system of $162 \times 162$ dimension is obtained. Its resolution leads to the distribution of magnetization in the three MTB (see Fig. 8 for induced magnetization in MTB2).

This result is equivalent to a 3-D line element FEM [1], but it is obtained with the resolution of a very small system.

\section{CONCLUSION}

A new method has been developed to compute stray fields created by wound rods subjected to external fields that can present rapid spatial variations. With respect to FEM, the matrix system is small and its resolution is fast and easy. The comparison with FEM shows a good agreement in the case of low permeabilities. For higher permeabilities, inaccuracies appear but they are of only $10 \%$, which can be satisfactory for some applications. Our approach, based on magnetization approach, could also take into account nonlinear law for materials.

\section{REFERENCES}

[1] X. Brunotte and G. Meunier, "Line element for efficient computation of the magnetic field created by thin iron plates," IEEE Trans. Magn., vol. 26, pp. 2196-2198, Sept. 1990.

[2] S. Ratnajeevan and H. Hoole, Computer Aided Analysis and Design of Electromagnetic Devices. New York: Elsevier, 1989.

[3] O. Chadebec, J.-L. Coulomb, V. Leconte, J. P. Bongiraud, and G. Cauffet, "Modeling of static magnetic anomaly created by iron plates," IEEE Trans. Magn., vol. 36, pp. 667-671, Oct. 2000.

[4] A. Vishnevski, I. Krasnov, and A. Lapokov, "Calculation of static magnetization for thin-walled constructions by boundary element method," IEEE Trans. Magn., vol. 29, pp. 2152-2155, Sept. 1993. 